\title{
Randomized phase II study of second-line chemotherapy with the best available 5-fluorouracil regimen versus weekly administration of paclitaxel in far advanced gastric cancer with severe peritoneal metastases refractory to 5-fluorouracil- containing regimens (JCOG0407)
}

\author{
Tomohiro Nishina' ${ }^{1}$ Narikazu Boku' ${ }^{2}$ Masahiro Gotoh ${ }^{3} \cdot$ Yasuhiro Shimada ${ }^{2}$ • \\ Yasuo Hamamoto $^{4}$ - Hirofumi Yasui ${ }^{5}$ Kensei Yamaguchi ${ }^{6}$ Hiroki Kawai ${ }^{7}$. \\ Norisuke Nakayama $^{8} \cdot$ Kenji Amagai $^{9}$ Junki Mizusawa ${ }^{10} \cdot$ Kenichi Nakamura $^{10}$. \\ Kuniaki Shirao ${ }^{11}$ - Atsushi Ohtsu ${ }^{12}$. The Gastrointestinal Oncology Study Group of the \\ Japan Clinical Oncology Group
}

Received: 15 June 2015/Accepted: 1 September 2015/Published online: 19 September 2015

(C) The International Gastric Cancer Association and The Japanese Gastric Cancer Association 2015

\begin{abstract}
Background This randomized phase II study compared weekly administration of paclitaxel (wPTX) with the best available 5-fluorouracil (5-FU) regimen as second-line treatment for advanced gastric cancer patients with severe peritoneal metastasis refractory to fluoropyrimidine.

Methods In the best available 5-FU arm, continuous infusion of 5 -FU $\left(800 \mathrm{mg} / \mathrm{m}^{2} /\right.$ day, days $1-5$, every 4 weeks) was given to patients with prior chemotherapy including bolus 5-FU, and methotrexate and 5-FU sequential bolus injection (methotrexate at $100 \mathrm{mg} / \mathrm{m}^{2}$ followed by bolus 5 -FU at $600 \mathrm{mg} / \mathrm{m}^{2}$ with leucovorin,
\end{abstract}

Presented at the 2010 Annual Meeting of the American Society of Clinical Oncology, Chicago, June 6, 2010 (abstract 4052).

Tomohiro Nishina

tnishina@shikoku-cc.go.jp

1 Department of Gastrointestinal Medical Oncology, Shikoku Cancer Center, 160 Minami-Umenomoto Kou, Matsuyama, Ehime 791-0280, Japan

2 Department of Gastrointestinal Medical Oncology, National Cancer Center Hospital, Tokyo, Japan

3 Cancer Chemotherapy Center, Osaka Medical College Hospital, Takatsuki, Japan

4 Department of Medical Oncology, Tochigi Cancer Center, Utsunomiya, Japan

5 Division of Gastrointestinal Oncology, Shizuoka Cancer Center, Sunto-gun, Japan

6 Division of Gastroenterology, Saitama Cancer Center, Kita-adachi-gun, Japan weekly) was given to those who had previously received continuous infusion of 5-FU or oral administration of fluoropyrimidine. In the wPTX arm, paclitaxel $\left(80 \mathrm{mg} / \mathrm{m}^{2}\right)$ was administered on days 1,8 , and 15 , every 4 weeks. This study adopted a screening design (one-sided $\alpha=30 \%$ ) with the primary end point of overall survival.

Results One hundred patients were randomized to the 5 -FU arm $(n=49)$ or the wPTX arm $(n=51)$. Although the median survival time was 7.7 months in both arms, the 2-year survival rates were $2.9 \%$ in the 5-FU arm and $9.1 \%$ in the wPTX arm [hazard ratio 0.89 (95\% confidence interval $0.57-1.38$ ), one-sided $p=0.298\}$. The median progression-free survival was longer with wPTX than with 5-FU [3.7 months vs 2.4 months; hazard ratio 0.58 (95\% confidence interval 0.38-0.88), one-sided

7 Department of Gastroenterology, Aichi Cancer Center Hospital, Nagoya, Japan

8 Department of Gastroenterology, Kanagawa Cancer Center, Yokohama, Japan

9 Department of Gastroenterology, Ibaraki Prefectural Central Hospital, Kasama, Japan

10 JCOG Data Center/Operations Office, Center for Research Administration and Support, National Cancer Center, Tokyo, Japan

11 Department of Medical Oncology and Hematology, Faculty of Medicine, Oita University, Yufu, Japan

12 Exploratory Oncology Research and Clinical Trial Center, National Cancer Center, Kashiwa, Japan 
$p=0.005]$. The incidences of grade 4 neutropenia, grade 3/4 febrile neutropenia, diarrhea, and treatment-related death were $6 \%, 4 \%, 10 \%$, and $2 \%$, respectively, in the 5-FU arm and $2 \%, 0 \%, 0 \%$, and $0 \%$, respectively, in the wPTX arm.

Conclusions As second-line chemotherapy, wPTX appears feasible and promising. This regimen can be included in a test arm in future phase III trials for treatment of advanced gastric cancer with severe peritoneal metastasis.

Keywords Phase II study · Paclitaxel · 5-Fluorouracil . Gastric cancer $\cdot$ Peritoneal metastasis

\section{Introduction}

Chemotherapy confers a survival benefit for advanced gastric cancer (AGC) patients over best supportive care [1-3], and the combination of fluoropyrimidines with platinum is recognized as the standard first-line chemotherapy [4-7]. It has recently been shown that second-line chemotherapy with irinotecan and taxanes prolongs survival.

Peritoneal metastasis (PM) occurs in more than $50 \%$ of patients with unresectable and recurrent gastric cancer through their clinical course, and it causes various complications, such as ascites, intestinal stenosis, hydronephrosis, and obstructive jaundice; the prognosis of patients with severe PM is dismal and generally worse than that of those without PM [8]. The Japan Clinical Oncology Group (JCOG) conducted a randomized phase III study (JCOG0106) of 5-fluorouracil (5-FU) continuous infusion (5-FUci) versus sequential methotrexate (MTX) and 5-FU therapy in patients having AGC with PM and concluded that 5-FUci, which is one of the most feasible regimens, is the standard first-line chemotherapy for such patients [9]. Many of these patients have some complications that adversely affect their general condition and, thereby, make it difficult to perform chemotherapy, especially after failure of first-line chemotherapy. However, in clinical practice, it is important to develop a feasible regimen of second-line chemotherapy for these patients.

Since 5-FU has a different mode of action when given by bolus or continuous infusion, and there appears to be incomplete cross-resistance between the methods [10], these two methods of 5-FU administration were often applied sequentially for first- and second-line chemotherapy [11]. Considering that monotherapy with 5-FU seems less toxic than other chemotherapy regimens available for AGC, 5-FU administered by a method different from that used for first-line chemotherapy can be considered one treatment option for second-line chemotherapy, especially for patients with severe PM. In fact, it was difficult to set a "no treatment" group as a control arm in Japan; therefore, 5-FU given by bolus or continuous infusion, which was different from the previous chemotherapy, was used in the control arm in this study.

On the other hand, several phase II trials of weekly administration of paclitaxel (wPTX) for treatment of AGC showed its promising efficacy for PM [12] and good feasibility [13-15], and complications due to PM would have a very small impact on the safety of wPTX $[12,13]$.

This randomized phase II study was conducted to compare wPTX with the best available 5-FU regimen as second-line treatment for AGC patients with severe PM refractory to 5-FU-containing regimens.

\section{Patients and methods}

\section{Study population}

This randomized, open-arm, multicenter phase II clinical trial was conducted at 24 institutions in Japan participating in the Gastrointestinal Oncology Group of the JCOG. The eligibility criteria were as follows: histologically proven gastric adenocarcinoma; unresectable or recurrent disease with PM diagnosed radiologically, such as intestinal stenosis on barium enema examination, peritoneal nodules, and/or ascites on computed tomography within 28 days before registration (histological confirmation of metastasis was not mandatory); age 20-75 years; Eastern Cooperative Oncology Group performance status of 2 or less; and one prior chemotherapy consisting of fluoropyrimidine that should have been completed at least 14 days before registration. In recurrent cases, patients whose recurrence was found within 24 weeks after the last administration of adjuvant chemotherapy consisting of orally administered fluoropyrimidine were eligible. In unresectable cases, patients who showed disease progression during or within 4 weeks after the last administration of fluoropyrimidinecontaining chemotherapy as first-line chemotherapy, which had continued for 4 weeks or longer, were eligible. Patients were ineligible if they received prior chemotherapy with taxanes, or 5-FU-containing regimens comprising both bolus and continuous infusion 5-FU, leucovorin with oxaliplatin (FOLFOX) or irinotecan (FOLFIRI). Further inclusion criteria were as follows: absence of prior radiation therapy; no symptomatic central nervous system metastases; preserved organ function (white blood cell count $3000-12,000 / \mathrm{mm}^{3}$; platelet count $10 \times 10^{4} / \mathrm{mm}^{3}$ or greater; aspartate aminotransferase and alanine aminotransferase concentrations of $100 \mathrm{U} / \mathrm{L}$ or lower; total bilirubin concentration $1.5 \mathrm{mg} / \mathrm{dL}$ or lower; creatinine concentration of $1.5 \mathrm{mg} / \mathrm{dL}$ or lower; C-reactive protein of $10 \mathrm{mg} / \mathrm{dL}$ or lower); and no transfusions within 21 days 
before entry. Patients with severe PM causing complete bowel obstruction or massive ascites occupying the whole abdominal cavity (from the bottom of the pelvis to just below the diaphragm) were excluded from the study.

All eligible patients provided their written informed consent to participate in this study. This study was approved by the Clinical Trial Review Committee of the JCOG and the institutional review board of each participating institution. This study was registered with the University Hospital Medical Information Network Clinical Trials Registry [http://www.umin.ac.jp/ctr/] (number C000000138).

\section{Randomization and masking}

After confirmation of the eligibility criteria by telephone or fax at the JCOG Data Center, the patients were randomized to either the best available 5-FU arm or the wPTX arm by the minimization method of balancing the arms according to institution and Eastern Cooperative Oncology Group performance status $(0,1$, or 2$)$. Neither the investigators nor the patients were blinded to treatment allocation.

\section{Treatment}

Patients who were entered in this trial had been treated either by continuous exposure to 5-FU through orally administered fluoropyrimidine or 5-FUci or by intermittent exposure through bolus administration of 5-FU in combination with MTX or leucovorin. "Best available 5-FU" in this study was therefore defined as MTX and 5-FU for those pretreated by continuous administration of 5-FU and as 5-FUci for those pretreated by intermittent administration.

The 5-FUci regimen was given as $800 \mathrm{mg} / \mathrm{m}^{2} /$ day, on days $1-5$, every 4 weeks, and the MTX and 5-FU regimen consisted of weekly MTX bolus infusion $\left(100 \mathrm{mg} / \mathrm{m}^{2} /\right.$ day, day 1$)$, followed by 5 -FU bolus infusion $\left(600 \mathrm{mg} / \mathrm{m}^{2} /\right.$ day, day 1) with a 3-h interval, and leucovorin given orally or by intravenous injection $\left(10 \mathrm{mg} / \mathrm{m}^{2}\right.$, repeated every $6 \mathrm{~h}$, days 2-3). Paclitaxel was given as a 1-h infusion $(80 \mathrm{mg} /$ $\mathrm{m}^{2} /$ day, days 1,8 , and 15 ), every 4 weeks. The dose of each drug was reduced according to predefined criteria. The protocol treatment was repeated until there was disease progression, there were unacceptable toxic effects, or the patient refused treatment.

\section{Evaluations}

Physical examinations and laboratory tests were repeated on each day of drug administration or at least once every 2 weeks. Tumor assessments by computed tomography covering the chest, abdomen, and pelvis were repeated every 2 months after randomization. No independent radiologic review was performed. All adverse events were graded according to the National Cancer Institute Common Terminology Criteria for Adverse Events, version 3.0. Investigators reported all serious adverse events classified according to the following three categories: (1) death within 30 days from the last administration of the protocol treatment regardless of cause; (2) treatment-related death; and (3) grade 4 nonhematological adverse events. The JCOG Data and Safety Monitoring Committee reviewed all serious adverse events to judge whether an adverse event was attributable to the protocol treatment.

\section{Data management and statistical analysis}

The primary end point was overall survival, which is more robust and suitable for efficacy evaluation than progression-free survival or response rates as it was anticipated that many of the participants in this trial would not have target lesions. Secondary end points were adverse events. Overall survival was measured from the date of randomization to the date of death from any cause. Surviving patients and patients lost to follow-up were censored at the date of last contact. Progression-free survival was measured from the date of randomization to the data of death from any cause or progression diagnosed radiologically or judged clinically.

The median survival time (MST) for "best available 5-FU" was assumed to be 4-6 months [16-18], and it was expected that wPTX would prolong survival by a median of $2 \pm 0.5$ months. The sample size was calculated to be 100 in total, with a one-sided alpha level of 0.3 and power of at least $85 \%$. The planned accrual period was 2 years, but the actual accrual speed was slower than planned. Thus, the protocol was revised to extend the accrual period to 3.5 years.

One interim analysis was conducted when 50 patients had been accrued. The alternative hypothesis, hazard ratio of 0.715 , was tested at the one-sided significance level of 0.05 to evaluate the futility of wPTX.

The primary analysis of overall survival was conducted with the stratified log-rank test, and the estimated hazard ratio was determined with a stratified Cox's proportional hazards model with performance status as a stratum. Other time-to-event end points were analyzed by the unstratified method. All analyses were performed on an intention-totreat basis with SAS version 9.1 (SAS Institute, Cary, NC, USA). Unless otherwise specified, $p$ values are presented as one-sided.

\section{Role of the funding source}

The sponsor of the study, the Ministry of Health, Labour and Welfare, Japan, had no role in study design, data 
collection, data analysis, data interpretation, or writing of the report. The Chair of the Gastrointestinal Study Group of the JCOG (N.B.) had full access to all data in the study and had final responsibility for the decision to submit the study for publication.

\section{Results}

\section{Study population}

Between July 2005 and December 2008, 100 patients were randomized from 24 institutions in Japan; 49 patients were allocated to the "best available 5-FU" arm (5-FUci for seven patients, MTX and 5-FU for 42 patients) and 51 patients were allocated to the wPTX arm. One patient was ineligible because the last prior chemotherapy was administered within 14 days before registration (Fig. 1). Baseline patient and disease characteristics were well balanced between the two treatment arms (Table 1). Almost all individuals had a performance status of 0 or 1 . More than $60 \%$ of patients had two or more metastatic lesions. Histologically diffuse-type tumors were dominant in both arms, and unresectable cases were more frequent than recurrent cases. With regard to the imaging diagnosis of PM, stenosis of the intestinal tract was commoner than ascites. In November 2009, the primary analysis was performed for all eligible patients on the basis of data obtained up to 11 months after the last patient had been enrolled.

\section{Safety}

Table 2 shows the adverse events recorded within 6 months after randomization. In the "best available 5-FU" arm, the grade 3 or 4 adverse events with frequencies higher than $10 \%$ were leukopenia $(10.2 \%)$, neutropenia $(28.6 \%)$, anemia $(12.2 \%)$, anorexia (14.3\%), infection with neutropenia $(10.2 \%)$, and diarrhea $(10.2 \%)$. In the wPTX arm, they were neutropenia $(11.8 \%)$ and anemia $(17.6 \%)$. Neutropenia, anorexia, and diarrhea were more frequent in the "best available 5-FU" arm than in the wPTX arm. Grade 3 or 4 febrile neutropenia was observed only in the "best available 5-FU" arm (4.1\%), and grade 3 or 4 sensory neuropathy was observed only in the wPTX arm $(3.9 \%)$. The reasons for stopping treatment were disease progression $(85 \%)$, toxicity $(8 \%)$, and patient refusal of treatment related to adverse events $(5 \%)$. The proportion of patients discontinuing protocol therapy because of toxicity was $10.2 \%$ in the "best available 5-FU" arm and $15.7 \%$ in the wPTX arm; wPTX was stopped in three patients because of cumulative toxicity for longer than 6 months after randomization, although it was associated with persisting efficacy.
The incidence of any serious adverse event was $6.1 \%$ in the "best available 5-FU" arm and $2.0 \%$ in the wPTX arm. The commonest serious adverse event was death within 30 days after the last treatment $(n=4)$. Treatmentrelated death occurred in one patient in the "best available 5-FU" arm; this patient died of pneumonia and disseminated intravascular coagulation.

\section{Efficacy}

At the time of analysis, 92 deaths $(93 \%)$ were observed. The 1-year overall survival rate was $27.1 \%$ in the "best available 5-FU" arm and $31.4 \%$ in the wPTX arm, and the 2-year survival rates were $2.9 \%$ and $9.1 \%$, respectively. The MST was 7.7 months [95\% confidence interval (CI), 6.7-9.0 months] in the "best available 5-FU" arm and 7.7 months (95\% CI 6.0-9.7 months) in the wPTX arm [hazard ratio (HR) 0.89 ; $95 \%$ CI $0.57-1.38$; one-sided $p=0.298$ ] (Fig. 2). The median progression-free survival time was 2.4 months (95\% CI 1.7-3.6 months) in the "best available 5-FU" arm and 3.7 months $(95 \% \mathrm{CI}$ 2.6-5.3 months) in the wPTX arm (HR 0.58; $95 \%$ CI $0.38-0.88$; one-sided $p=0.005$ ) (Fig. 3).

\section{Poststudy treatment}

Table 3 shows the details of poststudy treatment with the data obtained from 2 months of additional follow-up after primary analysis. Third-line chemotherapy was given to 42 patients ( $86 \%$ ) in the "best available 5-FU" arm and to 34 patients $(67 \%)$ in the wPTX arm. In the "best available 5-FU" arm, 37 patients (76 \%) received a taxane-containing regimen as third-line chemotherapy; 33 patients received a wPTX regimen.

\section{Discussion}

Recently, four randomized phase III trials that compared chemotherapy with best supportive care in the second-line setting for AGC were reported. The Arbeitsgemeinschaft Internistische Onkologie group trial [19] used irinotecan, the Korean group trial [20] used irinotecan or docetaxel, the COUGAR-02 trial used docetaxel [21], and the REGARD trial investigated ramucirumab [22] in the chemotherapy arm. These trials demonstrated a survival benefit when the drugs were used as second-line chemotherapy for AGC refractory to fluoropyrimidines and platinum. The West Japan Oncology Group (WJOG) 4007 trial was a phase III study conducted in Japan that compared irinotecan and wPTX in the second-line treatment of patients with AGC in which both treatment arms showed similar survival [23]. These results showed that second-line 
Fig. 1 CONSORT diagram. 5$F U$ 5-fluorouracil

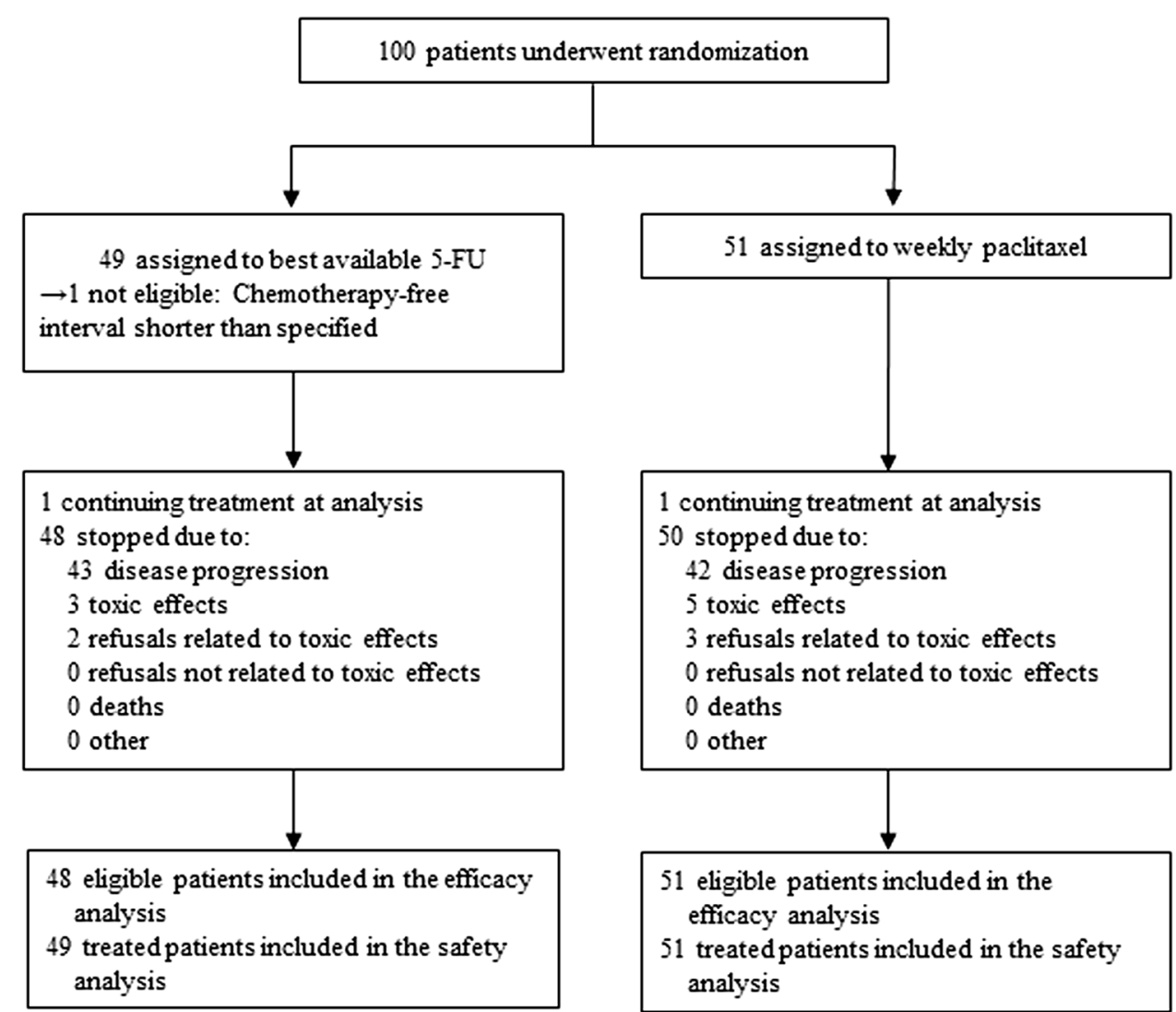

chemotherapy has a survival benefit for AGC patients, and combinations with irinotecan, docetaxel, wPTX, or ramucirumab are recognized as standard care after failure of combination therapy with fluoropyrimidine and platinum. However, these trials of second-line chemotherapy did not focus on patients with severe PM. Moreover, irinotecan is contraindicated for many patients with severe PM. This is the first randomized clinical trial comparing chemotherapeutic agents as second-line chemotherapy for AGC patients with severe PM. The results showed that the primary end point of this study was met from the statistical perspective $(p<0.3)$.

The first concern about chemotherapy for such patients is its safety, as the condition of gastric cancer patients with severe PM is generally poor, especially after failure of firstline chemotherapy. However, most patients in this study has a performance status of 0 or 1 , and the incidences of treatment-related serious adverse events in both arms were low (4\% in the "best available 5-FU" arm, $0 \%$ in the wPTX arm). The incidence of grade 3 or 4 neutropenia was $12 \%$ in the "best available 5-FU" arm, and febrile neutropenia was not observed in the wPTX arm. These results are comparable to those from a previous phase III study using wPTX in gastric cancer patients without severe PM [23]. The incidences of severe neutropenia and peripheral neuropathy in the wPTX arm in this study were less than those seen with 3-weekly administration of paclitaxel at a dose of $210 \mathrm{mg} / \mathrm{m}^{2}$ in patients with gastric cancer [24]. Moreover, severe gastrointestinal toxic effects were observed in only a few patients in the wPTX arm despite intestinal stenosis and/or ascites. As anticipated, wPTX was well tolerated in most patients with severe PM. Contrary to our assumption that "best available 5-FU" is less toxic than wPTX, the incidences of grade 3 or 4 neutropenia, febrile neutropenia, anorexia, and diarrhea were higher in the "best available 5-FU" arm than in the wPTX arm. These findings may have resulted from the high proportion of the bolus 5-FU regimen $(85.7 \%)$ in the "best available 5-FU" arm, considering that the hematological and intestinal toxic effects of bolus infusion of 5-FU are severer than those of 5-FUci [25]. Today, as orally administered fluoropyrimidine and 5-FUci play an important role in the first-line chemotherapy for AGC regardless of the presence of intestinal stenosis and/or ascites, bolus infusion of 5-FU is used for most cases as the "best available 5-FU" in second-line chemotherapy. Thus, it is considered that wPTX is more feasible than "best available (bolus) 5-FU'.

The next concern is the benefit of second-line chemotherapy for gastric cancer patients with severe PM 
Table 1 Patient and disease characteristics at study entry

\begin{tabular}{|c|c|c|}
\hline & "Best available 5-FU" arm $(n=49)$ & wPTX $\operatorname{arm}(n=51)$ \\
\hline Age (years), median/range & $59 / 30-74$ & $64 / 39-75$ \\
\hline Sex, male/female & $33 / 16$ & $36 / 15$ \\
\hline ECOG performance status, $0 / 1 / 2$ & $25 / 23 / 1$ & $27 / 22 / 2$ \\
\hline Macroscopic type $^{\mathrm{a}}, 0 / 1,2 / 3,4,5 /$ unknown & 2/8/38/1 & $1 / 9 / 39 / 2$ \\
\hline Histological type ${ }^{\mathrm{b}}$, intestinal/diffuse & $5 / 44$ & $4 / 47$ \\
\hline Surgery, unresectable/recurrent & $35 / 14$ & $30 / 21$ \\
\hline \multicolumn{3}{|l|}{ Previous chemotherapy (fluoropyrimidine) } \\
\hline 5-FU bolus & 7 & 9 \\
\hline 5-FU continuous infusion & 4 & 6 \\
\hline UFT & 3 & 1 \\
\hline S-1 & 33 & 32 \\
\hline Capecitabine & 2 & 3 \\
\hline \multicolumn{3}{|c|}{ Combined agents with fluoropyrimidine in previous chemotherapy } \\
\hline Cisplatin & 9 & 9 \\
\hline Methotrexate & 7 & 8 \\
\hline L-Leucovorin & 2 & 2 \\
\hline Irinotecan & 1 & 0 \\
\hline No. of metastatic sites, $1 / \geq 2$ & $18 / 31$ & $19 / 32$ \\
\hline \multicolumn{3}{|l|}{ Peritoneal metastasis } \\
\hline Intestinal stenosis ${ }^{\mathrm{c}}$, absent/present & $44 / 5$ & $47 / 4$ \\
\hline Ascites, absent/present & $9 / 40$ & $7 / 44$ \\
\hline
\end{tabular}

ECOG Eastern Cooperative Oncology Group, 5-FU 5-fluorouracil, UFT tegafur-uracil, wPTX weekly administration of paclitaxel

a Japanese classification of gastric carcinoma

b Lauren classification

c On barium enema

\begin{tabular}{|c|c|c|c|c|}
\hline \multirow[t]{2}{*}{ Adverse event } & \multicolumn{2}{|c|}{ "Best available 5-FU" arm $(n=49)$} & \multicolumn{2}{|c|}{ wPTX $\operatorname{arm}(n=51)$} \\
\hline & All grades $(\%)$ & Grade $3 / 4(\%)$ & All grades $(\%)$ & Grade $3 / 4(\%)$ \\
\hline Leukopenia & 67.3 & 10.2 & 86.3 & 3.9 \\
\hline Neutropenia & 69.4 & 28.6 & 70.6 & 11.8 \\
\hline Anemia & 75.5 & 12.2 & 82.4 & 17.6 \\
\hline Thrombocytopenia & 12.2 & 2.0 & 3.9 & 2.0 \\
\hline Febrile neutropenia & 4.1 & 4.1 & 0.0 & 0.0 \\
\hline Infection with neutropenia & 12.2 & 10.2 & 7.8 & 2.0 \\
\hline Hyponatremia & 38.8 & 4.1 & 27.5 & 0.0 \\
\hline Neuropathy (sensory) & 12.2 & 0.0 & 39.2 & 3.9 \\
\hline Anorexia & 71.4 & 14.3 & 41.2 & 2.0 \\
\hline Diarrhea & 46.9 & 10.2 & 27.5 & 0.0 \\
\hline Nausea & 53.1 & 6.1 & 27.5 & 0.0 \\
\hline Abdominal pain & 42.9 & 2.0 & 23.5 & 0.0 \\
\hline
\end{tabular}

5-FU 5-fluorouracil, $w P T X$ weekly administration of paclitaxel
Table 2 Adverse events (National Cancer Institute Common Terminology Criteria for Adverse Events, version 3.0) who have a dismal prognosis and are generally considered to be unfit for chemotherapy. The progression-free survival of the paclitaxel arm in this study was similar to that in the WJOG4007 trial [23] targeting patients without severe PM and longer than that of patients who received best supportive care in the REGARD trial [22] (median progression-free survival times of $3.7,3.4$, and 1.3 months, respectively). Although the MSTs of both arms (about 
Fig. 2 Overall survival (primary analysis). $C I$ confidence interval, 5- $\mathrm{FU}$ best available 5-fluorouracil regimen, $H R$ hazard ratio, $M$ months, $M S T$ median survival time, $w P T X$ weekly administration of paclitaxel, $1-y$ 1-year survival rate

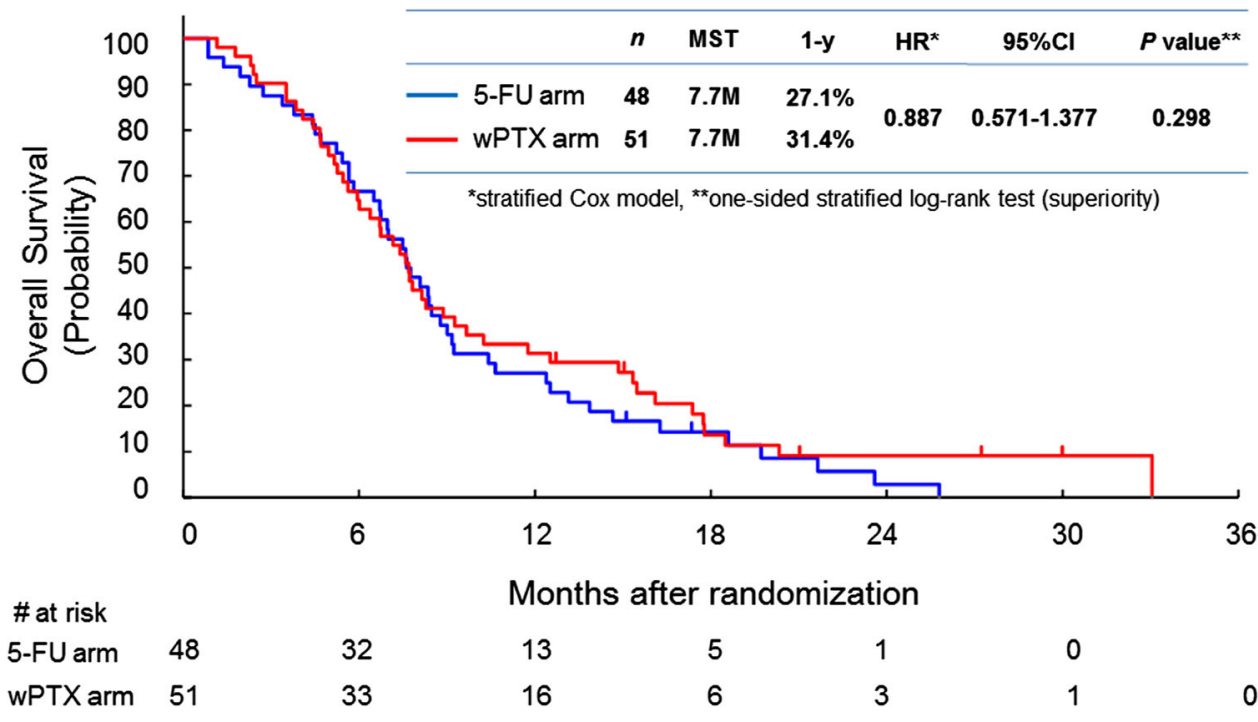

Fig. 3 Progression-free survival (exploratory analysis). $\mathrm{CI}$ confidence interval, 5-FU best available 5-fluorouracil regimen, $H R$ hazard ratio, $M$ months, $M S T$ median survival time, $w P T X$ weekly administration of paclitaxel, $1-y$ 1-year survival rate

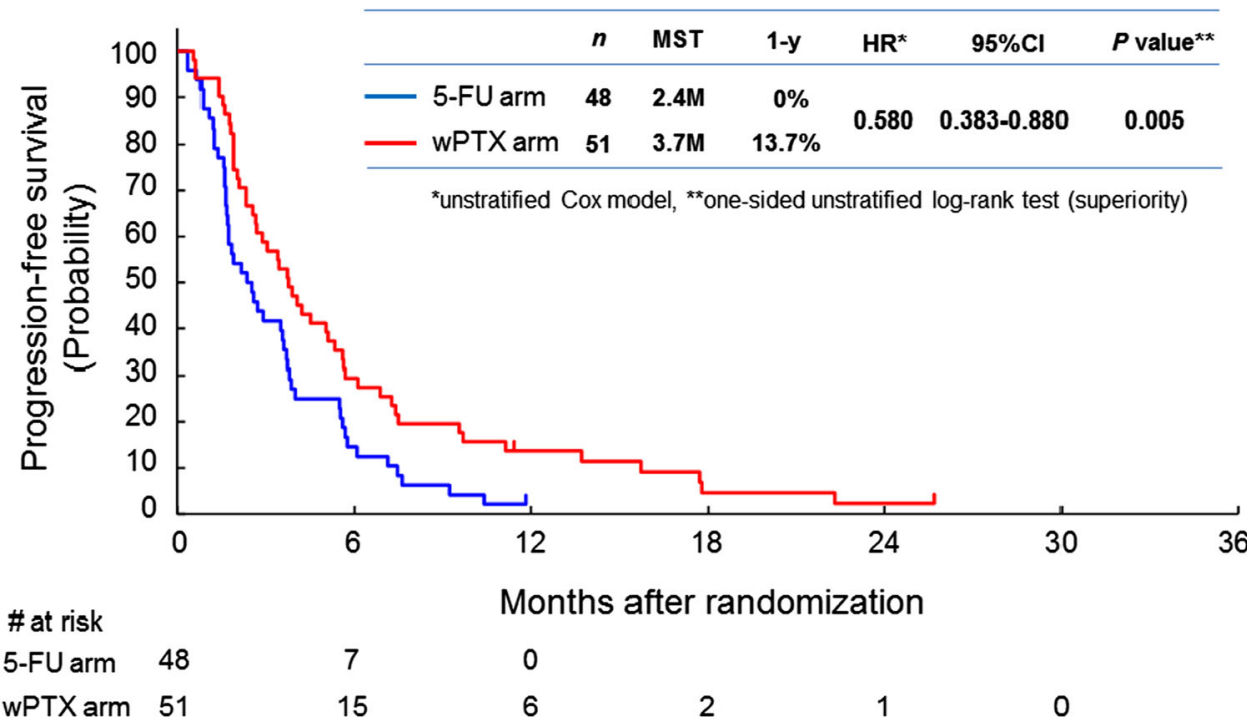

Table 3 Third-line treatment (proportion of crossover)

\begin{tabular}{lll}
\hline & "Best available 5-FU" arm $(n=49)$ & wPTX arm $(n=51)$ \\
\hline 3rd-line chemotherapy & 42 & 34 \\
wPTX & $33(67.3 \%)$ & - \\
MTX and 5-FU/5-FU continuous infusion & - & $13 / 1(27.5 \%)$ \\
Other & 9 & 20 \\
No 3rd-line chemotherapy & 6 & 16 \\
2nd-line chemotherapy continued & 1 & 1 \\
\hline
\end{tabular}

5-FU 5-fluorouracil, MTX methotrexate, wPTX weekly administration of paclitaxel

7 months) in the present study seemed to be shorter than the MST of the wPTX arm in the WJOG4007 trial [23] (9.8 months), they were longer than those of patients who received best supportive care in the other trials (2.5 months [19] and 3.8 months [20]), in which detailed information about PM was not available. Thus, second-line 
chemotherapy appears to have substantial activity contributing to survival prolongation even for gastric cancer patients with severe PM compared with best supportive care. However, one reason for the longer survival of the present patients compared with those in the previous reports could be that most of the patients in the present study had good performance status ( 0 or 1$)$. Thus, careful patient selection might be important for second-line chemotherapy in patients with severe PM.

At the time of planning this study, we anticipated that the MST of patients with severe PM was 4-5 months on the basis of previous reports [16-18]. Compared with our assumption about the MST in the "best available 5-FU" arm (4-6 months), the actual MST with "best available 5-FU" was much longer (7.7 months), whereas the MST of the wPTX arm was as long as expected (7.7 months). There were substantial imbalances in the third-line chemotherapy in this study. In particular, two thirds of the patients in the "best available 5-FU" arm received third-line chemotherapy with wPTX. Therefore, postprogression survival was analyzed to explore the impact of the third-line chemotherapy as poststudy treatment with the data obtained from additional follow-up after primary analysis, although this post hoc analysis was not specified in the protocol. Postprogression survival was longer in the "best available 5-FU" arm than in the wPTX arm (5.4 months vs 3.7 months, respectively). It has been reported that the efficacy of wPTX in third-line chemotherapy was as good as that in second-line chemotherapy [26]. The high proportion $(67 \%)$ of crossover treatment to wPTX in the patients in the "best available 5-FU" arm likely confounded the survival data, and this could explain the discrepancy between overall survival and progression-free survival. Therefore, one may speculate that wPTX may be active for gastric cancer patients with severe PM in any line of therapy.

There are some limitations in this clinical trial. First, the alpha error (0.3) was substantially higher than in usual randomized phase II trials, resulting in a very small sample size for this trial. Second, there was no confirmed evidence for the efficacy and safety of the "best available 5-FU" as the second-line chemotherapy when this study was being planned. In fact, the median progression-free survival in the "best available 5-FU" arm was as short as that of patients who received best supportive care in other clinical trials, and it was associated with more toxic effects than wPTX. The favorable overall survival time in the "best available 5-FU" arm might be due to subsequent chemotherapy, mainly with wPTX.

In conclusion, second-line chemotherapy may contribute to survival prolongation even for far advanced gastric cancer patients with severe PM refractory to 5-FU-containing regimens if they are in good medical condition, and
wPTX could be an option with feasible toxicity and promising efficacy for such patients. Paclitaxel administered weekly could be included in a future phase III trial.

Acknowledgments The authors dedicate this manuscript to Dr Hiroya Takiuchi, who unfortunately passed away in 2012. Without his great effort as the study coordinator, this study would not have been completed. The authors also thank Naoko Murata and Yuka Sakamoto for data management, Haruhiko Fukuda as a chair of the Japan Clinical Oncology Group Data Center supporting this study, and the Data and Safety Monitoring Committee and the Audit Committee of the Japan Clinical Oncology Group. This study was supported by Grants-in-Aid for Cancer Research (14S-3, 14S-4, 17S-3, 17S-5, 20S3, 20S-6, 26-A-4) and by Health and Labour Sciences Research Grants for Clinical Cancer Research (14-Gan-36, 17-Gan-008, 20-Gan-008) from the Ministry of Health, Labour and Welfare of Japan.

\section{Compliance with ethical standards}

Conflict of interest T.N. has received research funding from Taiho Pharmaceutical Co. Ltd. N.B. has received honoraria from Taiho Pharmaceutical Co. Ltd. N.B. has also received research funding from Chugai Pharmaceutical Co. Ltd. Y.S. has received research funding from Taiho Pharmaceutical Co. K.Y. has received honoraria from Merck Serono Co. Ltd., Bristol-Myers Squibb, and Takeda Pharmaceutical Co. Ltd. K.Y. has received research funding from Merck Serono Co., Ltd., Bristol-Myers Squibb, and Takeda Pharmaceutical Co. Ltd. K.A. has received research funding from Taiho Pharmaceutical Co. Ltd. K.S. has received research funding from Taiho Pharmaceutical Co. Ltd. All other authors declare that they have no conflict of interest.

Ethics All procedures followed were in accordance with the ethical standards of the responsible committee on human experimentation (institutional and national) and with the Helsinki Declaration of 1964 and later versions. Informed consent or a substitute for it was obtained from all patients for their being included in the study.

\section{References}

1. Glimelius B, Hoffman K, Haglund U, Nyrén O, Sjödén PO. Initial or delayed chemotherapy with best supportive care in advanced gastric cancer. Ann Oncol. 1994;5:189-90.

2. Pyrhönen S, Kuitunen T, Nyandoto P, Kouri M. Randomised comparison of fluorouracil, epidoxorubicin and methotrexate (FEMTX) plus supportive care with supportive care alone in patients with non-resectable gastric cancer. $\mathrm{Br} \mathrm{J}$ Cancer. 1995;71:587-91.

3. Wagner AD, Grothe W, Haerting J, Kleber G, Grothey A, Fleig WE. Chemotherapy in advanced gastric cancer: a systematic review and meta-analysis based on aggregate data. J Clin Oncol. 2006;24:2903-9.

4. Cunningham D, Starling N, Rao S, Iveson T, Nicolson M, Coxon $\mathrm{F}$, et al. Capecitabine and oxaliplatin for advanced esophagogastric cancer. N Engl J Med. 2008;358:36-46.

5. Koizumi W, Narahara H, Hara T, Takagane A, Akiya T, Takagi $\mathrm{M}$, et al. S-1 plus cisplatin versus S-1 alone for first-line treatment of advanced gastric cancer (SPIRITS trial): a phase III trial. Lancet Oncol. 2008;9:215-21.

6. Kang YK, Kang WK, Shin DB, Chen J, Xiong J, Wang J, et al. Capecitabine/cisplatin versus 5-fluorouracil/cisplatin as first-line 
therapy in patients with advanced gastric cancer: a randomised phase III noninferiority trial. Ann Oncol. 2009;20:666-73.

7. Ajani JA, Rodriguez W, Bodoky G, Moiseyenko V, Lichinitser $\mathrm{M}$, Gorbunova V, et al. Multicenter phase III comparison of cisplatin/S-1 with cisplatin/infusional fluorouracil in advanced gastric or gastroesophageal adenocarcinoma study: the FLAGS trial. J Clin Oncol. 2010;28:1547-53.

8. Fujimura T, Ishii K, Oyama K, Miyashita T, Kinami S, Ninomiya I, et al. A new scoring system for peritoneal metastasis in gastric cancer. Gastric Cancer. 2003;6:146-52.

9. Shirao K, Boku N, Yamada Y, Yamaguchi K, Doi T, Goto M, et al. Randomized phase III study of 5-fluorouracil continuous infusion vs. sequential methotrexate and 5-fluorouracil therapy in far advanced gastric cancer with peritoneal metastasis (JCOG0106). Jpn J Clin Oncol. 2013;43:972-80.

10. Hamaguchi T, Shirao K, Yamamichi N, Hyodo I, Koizumi W, Seki S, et al. A phase II study of sequential methotrexate and 5-fluorouracil chemotherapy in previously treated gastric cancer: a report from the Gastrointestinal Oncology Group of the Japan Clinical Oncology Group, JCOG 9207 trial. Jpn J Clin Oncol. 2008:38:432-7.

11. Kubota T, Watanabe M, Otani Y, Kitajima M, Fukushiuma M. Different pathways of 5-fluorouracil metabolism after continuous venous or bolus injection in patients with colon carcinoma: possible predictive value of thymidylate synthetase mRNA and ribonucleotide reductase for 5-fluorouracil sensitivity. Anticancer Res. 2002;22:3537-40.

12. Imamoto H, Oba K, Sakamoto J, Iishi H, Narahara H, Yumiba T, et al. Assessing clinical benefit response in the treatment of gastric malignant ascites with non-measurable lesions: a multicenter phase II trial of paclitaxel for malignant ascites secondary to advanced/recurrent gastric cancer. Gastric Cancer. 2011;14: 81-90.

13. Kadokura M, Iwasa S, Honma Y, Kato K, Hamaguchi T, Yamada $\mathrm{Y}$, et al. Weekly paclitaxel as second-line chemotherapy in Japanese patients with advanced gastric cancer. Anticancer Res. 2013;33:4547-52.

14. Kodera Y, Ito S, Mochizuki Y, Fujitake S, Koshikawa K, Kanyama Y, et al. A phase II study of weekly paclitaxel as second-line chemotherapy for advanced gastric cancer (CCOG0302 study). Anticancer Res. 2007;27:2667-71.

15. Emi Y, Yamamoto M, Takahashi I, Orita H, Kakeji Y, Kohnoe S, et al. Phase II study of weekly paclitaxel by one-hour infusion for advanced gastric cancer. Surg Today. 2008;38:1013-20.

16. Konishi T, Hiraishi M, Mafune K, Miyama T, Hirata T, Mori K, et al. Therapeutic efficacy and toxicity of sequential methotrexate and 5-fluorouracil in gastric cancer. Anticancer Res. 1994;14: $1277-9$.
17. Dickinson R, Presgrave P, Levi J, Milliken S, Woods R. Sequential moderate-dose methotrexate and 5-fluorouracil in advanced gastric adenocarcinoma. Cancer Chemother Pharmacol. 1989;24:67-8.

18. Yamao T, Shimada Y, Shirao K, Ohtsu A, Ikeda N, Hyodo I, et al. Phase II study of sequential methotrexate and 5-fluorouracil chemotherapy against peritoneally disseminated gastric cancer with malignant ascites: a report from the gastrointestinal oncology study group of the Japan Clinical Oncology Group, JCOG 9603 trial. Jpn J Clin Oncol. 2004;34:316-22.

19. Thuss-Patience PC, Kretzschmar A, Bichev D, Deist T, Hinke A, Breithaupt K, et al. Survival advantage for irinotecan versus best supportive care as second-line chemotherapy in gastric cancer-a randomised phase III study of the Arbeitsgemeinschaft Internistische Onkologie (AIO). Eur J Cancer. 2011;47:2306-14.

20. Kang JH, Lee SI, Lim DH, Park KW, Oh SY, Kwon HC, et al. Salvage chemotherapy for pretreated gastric cancer: a randomized phase III trial comparing chemotherapy plus best supportive care with best supportive care alone. J Clin Oncol. 2012;30: 1513-8.

21. Ford HE, Marshall A, Bridgewater JA, Janowitz T, Coxon FY, Wadsley J, et al. Docetaxel versus active symptom control for refractory oesophagogastric adenocarcinoma (COUGAR-02): an open-label, phase 3 randomised controlled trial. Lancet Oncol. 2014; $15: 78-86$.

22. Fuchs CS, Tomasek J, Yong CJ, Dumitru F, Passalacqua R, Goswami C, et al. Ramucirumab monotherapy for previously treated advanced gastric or gastro-oesophageal junction adenocarcinoma (REGARD): an international, randomised, multicentre, placebo-controlled, phase 3 trial. Lancet. 2014;383:31-9.

23. Hironaka S, Ueda S, Yasui H, Nishina T, Tsuda M, Tsumura T, et al. Randomized, open-label, phase III study comparing irinotecan with paclitaxel in patients with advanced gastric cancer without severe peritoneal metastasis after failure of prior combination chemotherapy using fluoropyrimidine plus platinum: WJOG 4007 trial. J Clin Oncol. 2013;10:4438-44.

24. Yamaguchi K, Tada M, Horikoshi N, Otani T, Takiuchi H, Saitoh $\mathrm{S}$, et al. Phase II study of paclitaxel with 3-h infusion in patients with advanced gastric cancer. Gastric Cancer. 2002;5:90-5.

25. Delfino A, Petrelli N. Meta-analysis of randomized trials testing the biochemical modulation of fluorouracil by methotrexate in metastatic colorectal cancer. Advanced colorectal cancer metaanalysis project. J Clin Oncol. 1994;12:960-9.

26. Shimoyama R, Yasui H, Boku N, Onozawa Y, Hironaka S, Fukutomi A, et al. Weekly paclitaxel for heavily treated advanced or recurrent gastric cancer refractory to fluorouracil, irinotecan, and cisplatin. Gastric Cancer. 2009;12:206-11. 